\title{
MECHANISMS OF CELL RESISTANCE IN GLIOBLASTOMA MULTIFORME
}

\author{
Victoria Sarafian ${ }^{1}$, Ilian Koev², Dmitrii Staykov ${ }^{3}$ \\ ${ }^{1}$ Department of Biology; \\ ${ }^{2}$ Department of Neurosurgery, \\ ${ }^{3}$ Department of General and Clinical Pathology \\ Medical University-Plovdiv, Bulgaria
}

\section{SUMMARY}

Glioblastoma multiforme is the most common brain tumor in adults. It is characterized by a rapid clinical course and extremely unfavorable prognosis. The etiology and the molecular pathogenetic mechanisms are not entirely clear yet. Active proliferation, resistance to apoptosis and high angiogenicity are basic factors limiting the effect of standard therapy.

A significant problem is the resistance of glioblastoma cells to apoptosis induced by most of antitumor drugs and radiotherapy. The functional activity of several tumorsuppressor genes is inhibited. The antiapoptotic effect of the normal brain matrix and the altered expression of integrin $\mathrm{aVb} 3$ act as main regulators of angiogenesis, invasion and proliferation of glioblastoma cells.

Therapeutic strategies are based on cellular and molecular mechanisms leading to: activation of apoptosis, inhibition of growth factors and receptors, and blocking of angiogenesis. Crucial moment in modern therapy is the activation of autophagy. Its effectiveness depends on the expression level of genes mTOR and MTMG. The most promising approach is the complex one combining surgery, radiotherapy and targeted molecular therapy directed simultaneously to different cellular pathogenetic mechanisms. resistance

Key words: glioblastoma, apoptosis, autophagy,

\section{INTRODUCTION}

Glioblastoma multiforme is the highest grade glioma tumor (grade IV) and is the most malignant form of astrocytomas. The unfavourable prognosis, short survival time and resistance to therapy render this tumor as one of the most devastating malignancies known. It is a vastly aggressive tumor charaterized by marked angiogenesis and extensive invasion into the normal parenchyma. There are no efficient therapeutic approaches with definite effect yet (1). Surgical removal, chemotherapy and radiotherapy improve survival for a short period after which the tumor relapses. It is clear now that glioblastoma cells exhibit intrinsic mechanisms rendering them resistant to all kinds of therapies available.

\section{Biological and morphological characteristics}

Glioblastoma is presented by a central tumor with rapid proliferation with invasive cells with down-regulated proliferation and high motility ensuring the occurrence of secondary tumors elsewhere. Glioblastoma cells show high intra- and intertumoral heterogeneity and resistance to apoptosis due to the deletion of tumor suppressor genes PTEN (noted in $30-40 \%$ of the cases) and p53 (in 25-35\%). The high proliferation rate results from the amplification of growth factor receptor genes EGFR (in $85 \%$ of the tumors) and PDGFR $(2,3)$. All these characteristics together with the presence of massive necrosis and high angiogeneity within the tumor contribute to its extreme invasiveness.

\section{Resistance to cell death}

It is known since 1949 that neurons undergo massive programmed cell death (apoptosis) during normal neurogenesis. This fact was shown by Viktor Hamburger and R. Levi-Montalcini and resulted in the discovery of the first growth factor - NGF (nerve growth factor). Synaptogenesis is a perinatal process during which $20-80 \%$ of neurons generated in the course of embryonic development die before adult age due to differences in number, defects, competition for neurotrophic factors, altered balance between activating and inhibiting neurotransmitters, etc. (4).

Cells die by two means: accidentally: by necrosis - due to acute mechanic or toxic factors (ionic transport, energy metabolism, $\mathrm{pH}$, ishemia) or following a genetic program: by apoptosis (programmed cell death type I) - genetically determined suicide with defined stages and typical morphology and by autophagy (programmed cell death type II) - lysosome-mediated degradation of proteins and cellular organelles.

Apoptosis is vitally important for normal development, but it also destroys cells threatening the integrity and development of the body like tumor cells. It is initiated either by external activation signals ("death receptors" - TNFR, Fas /CD95/) or by internal signals within the cell. The process is driven by the cascade activation of executor caspases followed by proteolysis of structural cytoplasmic proteins, 
chromatin degradation and finally, cell death. It is suggested that alterations in the apoptotic pathways in gliomas are important modulators of both gliomagenesis and response to therapy (5).

A key factor for the cellular homeostasis is the balance between proapoptotic (p53, PTEN, bax) and antiapoptotic (bcl2 ) genes and molecules. The expression of proliferative markers like Ki-67, PCNA, EGFR and Cyclin D1 is also of crucial significance.

The cells in the central nervous system exhibit differences in their resistance to apoptosis. The most vulnerable ones are the neurons, followed by oligodendrocytes and astrocytes, endothelial cells and microglia being the most resistant (6 ). During malignant progression most of these cell types acquire high resistance to apoptosis-inducing agents and procedures. The migrating cells in marginal zones of glioblastoma multiforme are less sensitive to apoptosis which results in frequent relapses (7). The antiapoptotic effect of the normal brain matrix and the interactions between glioma cells and the extracellular matrix also plays and important role. There is an inverse correlation between the increased migration and decreased sensitivity to apoptosis. This phenomenon is due to the enhanced expression of integrin $\mathrm{aVb} 3$ in marginal infiltrating zones where it serves as a basic regulator of angiogenesis, invasion and proliferation (3). Recent therapies imply antagonists of integrin aVb3 (Vitaxin) as means to inhibit angiogenesis and augment apoptosis in glioblastoma.

It is shown that glioblastoma cells carry mutations that inactivate apoptotic pathways thus necrosis could also represent an alternative pathway for tumor cells to be eliminated (7).

It is assumed that the higher apoptotic indexes in glioblastoma compared to astrocytoma result from higher proliferative activity and more prominent hypoxia (8). It is also suggested that the efficacy of radiotherapy may at least partially be mediated by an anti-angiogenic mechanism involving endothelial cell apoptosis.

It is now believed that cell resistance in glioblastoma is due to more than one gene or protein and multiple cell pathways are implicated. The pitfalls in therapy are also caused by the fact that activators of apoptosis and inhibitors of angiogenesis act not only in the central nervous system but in other organs too.

The biological characteristics of gliobastoma cells and the corresponding therapeutic approaches are summarized on Figure 1.

\section{Autophagy as a tool to fight glioblastoma}

Recently, programmed cell death type II - autophagy (caspase independent cell death) is accepted to act as a backup mechanism when apoptosis is inhibited (7). It operates as a protective mechanism against stress conditions as low oxygen and/or nutrient levels - circumstances often observed in glioblastoma. The proautophagic drugs are considered as the most efficient ones and are regarded as the Trojan horse in the battle against glioblastoma (9).

mTOR (mammalian target of rapamycin) is a kinase that normally inhibits apoptosis and autophagy and is active in sufficient nutrients supply within the cell. The activity of the mTOR pathway is often constitutively upregulated in tumors as a result of excessive stimulation by growth factor receptors.

Rapamycin is a drug produced by Streptomyces hygroscopicus and acts as a blocker of mTOR and thus as an activator of autophagy. mTOR inhibitors sensitize tumor cells to DNA-damaging agents in vitro (10). In glioblastoma an overactivation of $\mathrm{mTOR}$ is registered that leads to increased resistance to the proapoptotic effect of activators of both apoptosis and autophagy. It is believed that by inhibition of mTOR apoptosis-resistant migrating glioblastoma cells might be destroyed (11).

A promising drug is Temozolomide (Temodal) - a proautophagic cytotoxic substance that acts as an oral alkylating agent forming methylguanosine that pairs with thymine in DNA of tumor cells. As a result DNA replication is blocked and the cell cycle stops in phase G2/M (12). Part of its cytotoxic activity is exerted through pro-autophagic processes and also through the induction of apoptosis (12). Apoptosis can begin with autophagy and autophagy can end with apoptosis. Autophagy can therefore be seen as a backup mechanism that comes into action when apoptosis is inhibited (13).

Temodal activates autophagy but its benefits depend on the epigenetic silencing of the MTMG gene. MTMG gene (O-6-methylguanine-DNA methyltransferase) is a DNA reparative enzyme that removes alkyl groups from the O-6 position of guanine. Individual sensitivity to Temodal depends on the inactivation (methylation) of the MTMG gene (14). The lower the gene activity the higher the effect of Temodal treatment. Although proautophagic drugs are considered as the most efficient ones nowadays their activity is individual and might be possibly predicted by the examination of the genetic profile of the patient - MGMT gene methylation and mTOR expression.

\section{CONCLUSION}

The unfavourable prognosis, short survival time and resistance to therapy portray glioblastoma multiforme as one of the most destructive neoplasias known. The modern and promising approach is the complex one combining surgery, radiotherapy and targeted molecular therapy directed simultaneously to different cellular pathogenetic mechanisms. If apoptosis, autophagy and angiogenesis are targeted together the therapeutic efficiency could be increased, the toxicity decreased and the life span of patients with glioblastoma multiforme possibly prolonged. 


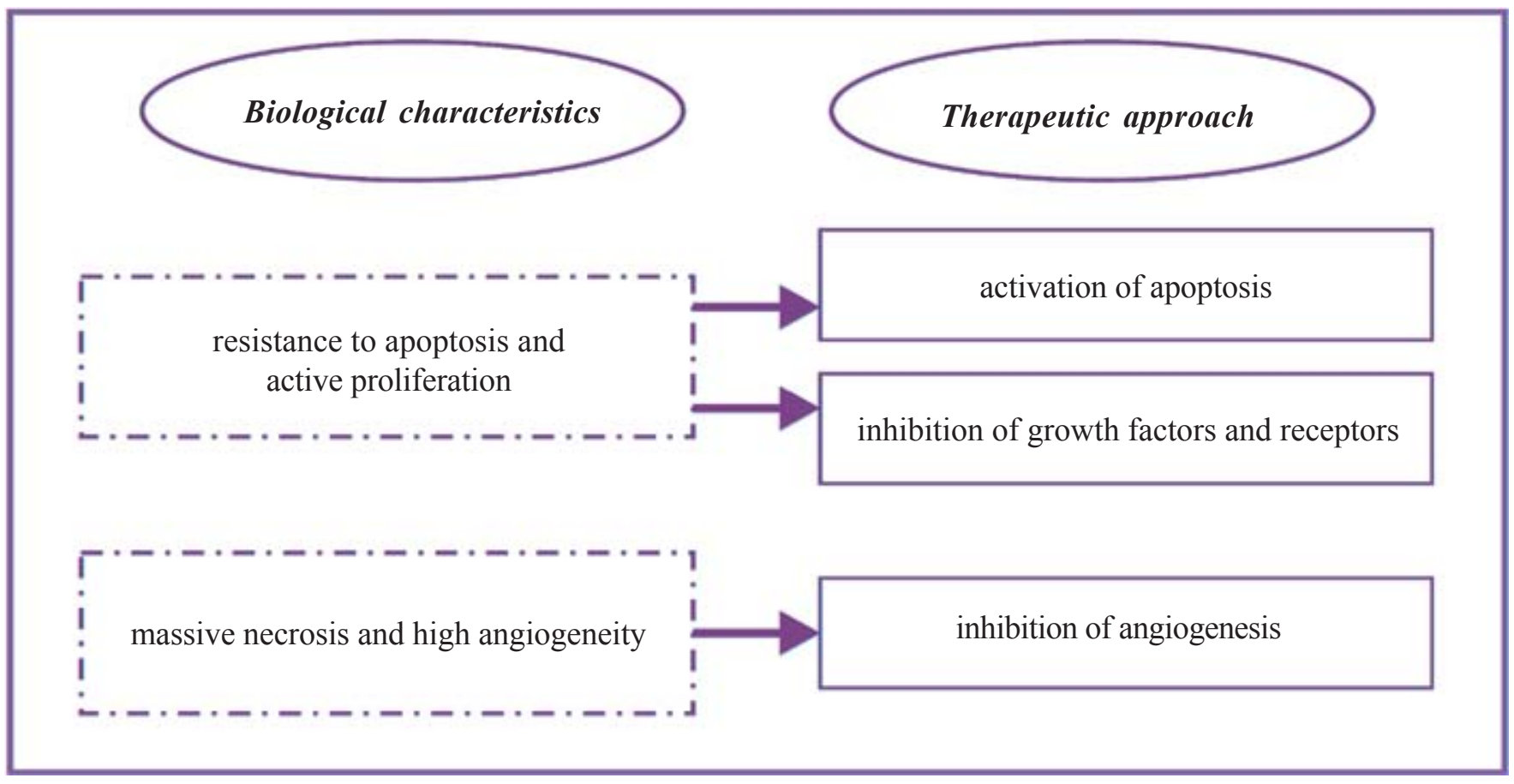

Figure 1. Biological characteristics and related therapeutic approaches in glioblastoma multiforme.

Acknowledgement. The study is supported by grant HO-01/09 from Medical University-Plovdiv.

\section{REFERENCES:}

1. Sathornsumetee S, Rich JN. New approaches to primary brain tumor treatment. Anticancer Drugs, 2006; 17: 1003-1016.

2. Royds JA, Iacopetta B. p53 and disease: when the guardian angel fails. Cell Death Different., 2006; 13: 1017-1026.

3. Bogler O, Mikkelsen T. Angiogenesis and apoptosis in glioma: two arenas for promising new therapies. J. Cell. Biochem., 2005; 96: 16-24.

4. Deshmukh M, Johnson E. Programmed cell death in neurons: focus on the pathway of nerve growth factordeprivation-induced death of sympathetic neurons. Mol. Pharmacol., 1997; 51: 897906.

5. Ziegler D, Kung A, Kieran M. Antiapoptosis mechanisms in malignant gliomas. J. CLin. Oncol., 2008; 3: 493-500.
6. Salgado S. Apoptotic and antiapoptotic mechanisms in neurons and glial cells after damage of immature brain. $\mathrm{PhD}$ thesis, 2007, p.19-21.

7. Lefrank F, Brotchi J, Kiss R. Possible future issues in the treatment of glioblastomas: special emphasis on cell migration and the resistance of migrating glioblastoma cells to apoptosis. J. CLin. Oncol., 2005; 23: 2411-2422.

8. Steinbach J, Weller M. Apoptosis in gliomas: molecular mechanisms and therapeutic implications. J NeuroOncol., 2004; 70: 245-254.

9. Lefrank F, Kiss R. Autophagy, the Trojan horse to combat glioblastomas. Neurosurg. Focus, 2006; 20: E1-7.

10. Meric-Bernstam F, GonzalezAngulo AM. Targeting the mTOR signaling network for cancer therapy. J. Clin. Oncol.,
2009; 1: 227822-87.

11. Omuro AM, Faivre S, Raymond E. Lessons learned in the development of targeted therapy for malignant gliomas. Mol. Cancer Ther., 2007; 6:1909-1919.

12. Lefranc F, Rynkowski M, DeWitte $\mathrm{O}$, Kiss R. Present and potential future adjuvant issues in high-grade astrocytic glioma treatment. Adv. Tech. Stand. Neurosurg., 2009;34:3-35.

13. Lefrank F, Facchini V, Kiss R. Proautophagic drugs: a novel means to combat apoptosis-resistant cancers, with a special emphasis on glioblastomas. The Oncologist, 2007; 12: 1395-1403.

14. Tentori L, Graziani G. Recent approaches to improve the antitumor efficacy of temozolomide. Curr. Med. Chem., 2009; 16: 245-257.

\section{Address for correspondence: \\ Dr Victoria Sarafian \\ Department of Biology, Medical University - Plovdiv \\ 15a, Vasil Aprilov Blvd., 4000 Plovdiv, Bulgaria \\ Tel. +359 32/602 531; 265 986; E-mail: sarafian@abv.bg}

